\title{
Multi-sensory training and wrist fractures: a randomized, controlled trial
}

\author{
Bergthora Baldursdottir ${ }^{1,2,3}$ (i) $\cdot$ Susan L. Whitney ${ }^{4} \cdot$ Alfons Ramel $^{2,5} \cdot$ Palmi V. Jonsson $^{1,2} \cdot$ Brynjolfur Mogensen $^{1}$. \\ Hannes Petersen ${ }^{1,6}$. Ella K. Kristinsdottir ${ }^{1}$
}

Received: 31 July 2018 / Accepted: 29 January 2019 / Published online: 11 February 2019

(c) The Author(s) 2019

\begin{abstract}
Background Asymmetric vestibular function, decreased plantar sensation, postural control and functional ability have been associated with fall-related wrist fractures.

Objective To investigate whether multi-sensory training (MST) improves postural control, vestibular function, foot sensation and functional ability among people with fall-related wrist fractures compared to wrist stabilization training (WT).

Methods This was an assessor-blinded, randomized controlled trial. Ninety-eight participants, age 50-75 years, were randomized to MST or WT. Pre- and post-training measurements: Head Shake Test (HST), Video-Head Impulse Test (vHIT), Semmes-Weinstein Monofilaments (SWF), Biothesiometer (BT), Sensory Organization Test (SOT), 10-m Walk Test (10MWT), Five Times Sit to Stand Test (FTSTS), Activities-Specific Balance Confidence (ABC) and Dizziness Handicap Inventory Scales (DHI). The training period was 12 weeks, with six supervised sessions by a physical therapist and daily home exercises for both groups.

Results There were significant endpoint differences in SOT $(p=0.01)$ between the two groups, in favor of the MST group, but no changes were seen in other outcome variables. Subgroup analysis with participants below normal baseline SOT composite scores indicated that the MST was more effective in improving 10MWT fast $(p=0.04)$, FTSTS $(p=0.04)$, SWF $(p=0.04)$ and SOT scores $(p=0.04)$ than the WT.

Conclusions MST improves postural control among people with a fall-related wrist fracture. The results further suggest that the program is more effective for those with SOT balance scores below age-related norms.
\end{abstract}

Keywords Fracture $\cdot$ Rehabilitation $\cdot$ Wrist $\cdot$ Sensation $\cdot$ Exercise

Bergthora Baldursdottir

bergbald@landspitali.is

1 Faculty of Medicine, University of Iceland, Reykjavik, Iceland

2 The Icelandic Gerontological Research Institute, Landspitali University Hospital, Reykjavik, Iceland

3 Department of Physical Therapy, Landspitali University Hospital, Tungata 26, 101 Reykjavik, Iceland

4 Department of Physical Therapy, University of Pittsburgh, Pittsburgh, USA

5 Faculty of Food Science and Nutrition, University of Iceland, Reykjavik, Iceland

6 Akureyri Hospital, Akureyri, Iceland

\section{Introduction}

Postural instability and falls are one of the major health concerns associated with increasing age. About one-third of people aged 65 and over fall each year and the incidence of falls doubles every 5 years thereafter [1]. Injuries and fractures are common consequences of falls. Wrist fracture (distal forearm fracture) has been reported as the most common injury in people between 65 and 74 years of age, attending an orthopedic emergency clinic after a fall [2]. Wrist fractures have also been shown to be a strong predictor of future fracture risk [3] and are often a precursor to hip fractures [4], which results in increased health costs, decreased quality of life and even death [5].

The most common profile of a patients affected by a wrist fracture is a functionally independent woman younger than 75 years of age [6]. Although the majority of wrist fracture 
subjects are apparently healthy, many of them exhibit risk factors for new falls and fractures. These can be regular medications [7], functional decline [6], history of previous falls and fractures [7], as well as asymmetric vestibular function $[8,9]$. In a recently published case-control study, postural control, plantar sensation, vestibular and physical functions were significantly worse among subjects having sustained a wrist fracture than healthy controls [10]. Furthermore, asymmetric vestibular function and decreased plantar pressure sensation showed the strongest associations with a fallrelated wrist fracture [10]. Some of these variables, such as vestibular function, postural control and physical function, can be enhanced by rehabilitation.

Group sessions with vestibular rehabilitation have reduced the incident of vestibular asymmetry among elderly people with wrist fracture [11]. Vestibular rehabilitation consists of balance exercises and the incorporation of head movements that may provoke dizziness. Symptoms are generated by using exercises comprising a sequence of eye, head and body movements of increasing difficulty [12, 13]. Multi-sensory exercises are characteristically defined as exercises that selectively stimulate and manipulate all the three afferent sensory systems including vestibular, visual and somatosensory pathways [14-18]. Hu and Wollacott, reported that multi-sensory balance training designed to improve intersensory interaction, improved balance performance in healthy older adults [14] and optimized the muscle and movement characteristics among the participants [15]. Multi-sensory training directed at improving function of the sensory systems has improved functional mobility [16] and reduced body sway in older adults living in the community [17]. A pilot study on the efficacy of a new multisensory balance training, "The Reykjavik model", consisting of combined mechano- and proprioceptive, vestibular and fall-prevention training, demonstrated that post-training, postural control, functional ability and confidence during daily activities improved among frail old people with a history of multiple falls [18].

Ongoing problems after a wrist fracture can encompass stiffness, pain and muscle weakness, which can lead to difficulties completing everyday functional tasks [19]. Physiotherapy usually consists of exercises for range of motion (ROM) and muscle strength to improve pain, range of motion, grip strength and activity in this population [20]. In the current emergency care settings in Iceland, people who have sustained a fall-related wrist fracture receive treatment for the fracture. When the cast has been removed ( $\sim 5-6$ weeks post-fracture), they are offered to participate in group training sessions with the aim to improve movement and strength in the wrist. Patients are referred to individual physical therapy sessions if their condition (range of motion restrictions and/or complex regional pain syndrome) requires further intervention. However, they are not routinely screened for falls and fracture risk nor offered an exercise program to improve or maintain balance control to reduce the risk of future falls.

The aim of the present study was to investigate whether, multi-sensory training (MST) "The Reykjavik model" improves postural control, vestibular function, foot sensation and functional ability among people with fall-related wrist fractures compared with those receiving wrist stabilization training (WT). Additionally, we wanted to investigate whether potential changes are affected by baseline balance control.

\section{Methods}

\section{Design overview}

This study was a randomized, controlled trial. All participants underwent baseline measurements within a week before intervention started. They were randomly assigned to one of two study arms: (1) intervention group=MST, and (2) control group $=$ WT. Participants in both groups attended six treatment sessions (30 min each) during a 3 -month period, which were supervised by a physical therapist (PT). In the first two training sessions, additional $30 \mathrm{~min}$ were allocated to the participants in both groups, so they could familiarize themselves with the proposed exercises. Participants in both groups further received a written exercise program that was to be performed daily at home. Duration of home exercises was a minimum of $15 \mathrm{~min}$, without upper time limits in both groups. The participants kept a home exercise diary during the training period. Outcome measurements were repeated within a week after the last training session.

\section{Setting and participants}

Ninety-eight individuals (mean age 61.9 \pm 7.1 ; range 50-75; females $=85$, males $=13$ ), who had previously sustained a fall-related wrist fracture, participated in this trial. They were identified from medical records at the Emergency Department of the Landspitali University Hospital in Reykjavik, Iceland and screened for eligibility from a total of 440 consecutive patients during a 12-month period. Enrolment commenced in May 2015 and ceased in May 2016. Subjects were recruited for the study 2-5 months after the fracture by an invitation letter, followed up by a phone call. Exclusion criteria were diagnosis of a degenerative CNS disease, such as Parkinson's, Alzheimer's or other diseases possibly impairing mobility or cognitive function. The study received permission from the Icelandic National Bioethics Committee (VSNb2013110036/03.11) and was conducted in accordance with the ethical standards laid down in the 1964 Declaration of Helsinki and its later amendments. The 
subjects provided informed written consent before participation in the study. A flow chart of study participants is shown in Fig. 1.

\section{Sample size}

The recruitment target was 90 participants. Sample size calculations were based on previous work by Herbert et al. [21], where they used composite scores from the sensory organization test as the primary outcome measure. With a power of $80 \%$ and significance level of 5\%, 36 people were required in each group in order to detect a mean difference of 10 SOT composite score assuming a standard deviation of 15 . The sample size was increased to 47 participants per group to allow for a $30 \%$ loss due to dropouts.

\section{Randomization and interventions}

After recruitment and baseline measurements, participants were randomized using a computer-created random number list and sealed envelopes.

\section{Intervention: multi-sensory training (MST), "the Reykjavik model"}

The exercises were performed barefoot on firm and soft surfaces, during quiet stance and movements. Throughout all the exercises, the subjects' attention was directed at weight distribution on the soles of the feet to recognize and control the position and movements of the body. They were also encouraged to be aware of their postural control pattern and

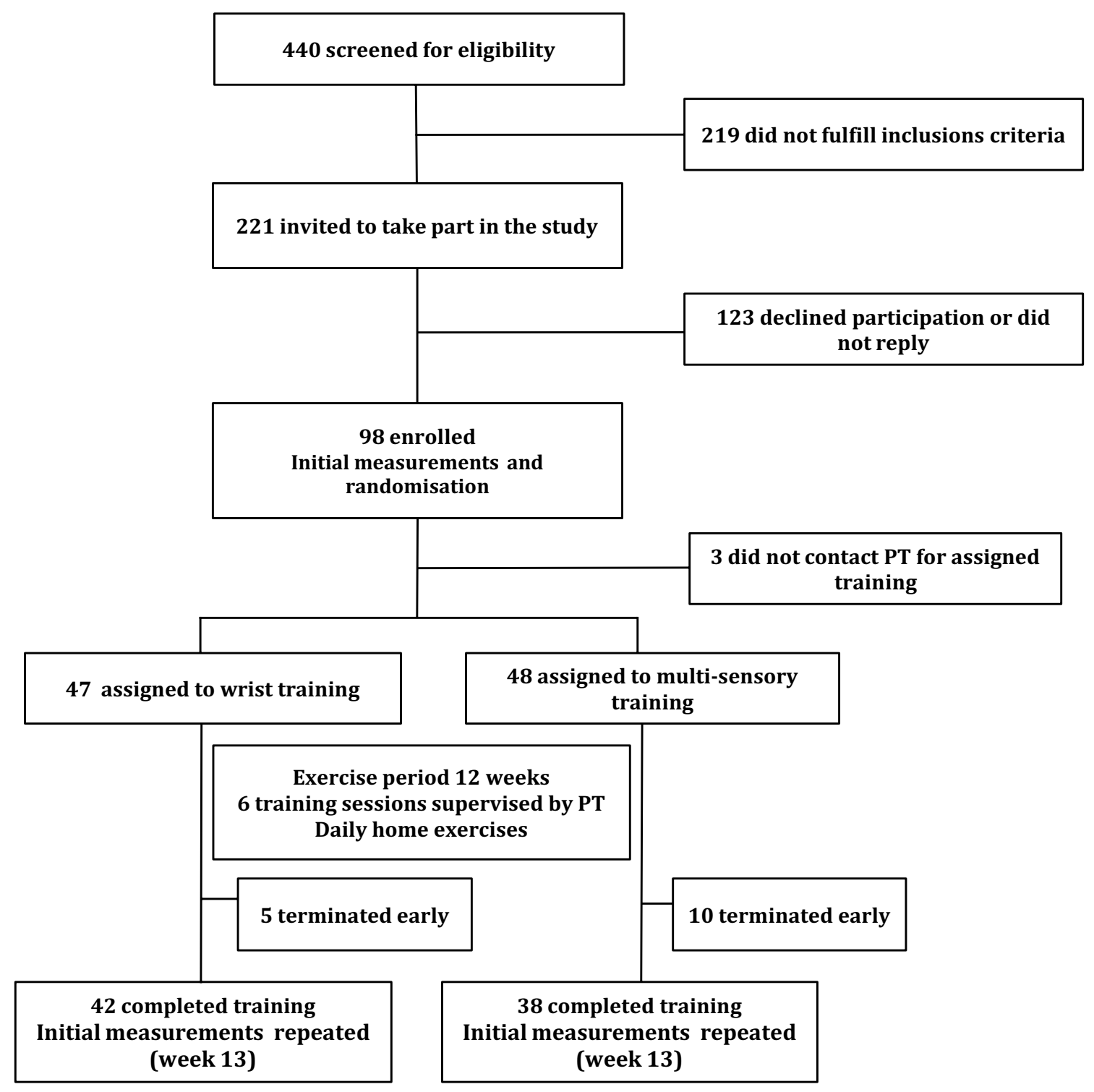

Fig. 1 Study flow chart 
taught to readjust their posture with slow smooth corrective motions at the ankles and avoid using high-frequency movements at the hips and upper body. The participants were discouraged to use their hands or body for external support. Control of posture was practiced during head movements in different directions, with eyes open, closed and during fixation of gaze. The subjects were taught how to react to sudden balance disturbances by taking a step to hinder falling and use stepping reactions when their stability was challenged by a manual push in different directions. Exercises were chosen from a list (Appendix 1) according to the main balance weaknesses of each subject, tailored close to the limits of capability, and progressed in the supervised sessions according to their improvements. The participants were instructed to perform the home exercises as well in barefoot, focus on weight distribution on the soles of the feet in all the exercises and performed the exercises until symptoms of dizziness and/or unsteadiness were provoked; then to take a short break to let the symptoms subside and then continue with the home exercise program. Examples of the MST home exercises performed in the present study can be viewed at: https://vimeo.com/252733777/c6eef955ad.

A detailed description of the training has previously been published [18] and the exercise program is demonstrated at: https://vimeo.com/album/4948077.

\section{Control group: wrist stabilization training (WT)}

The WT comprised a set of strengthening and coordination exercises for the fractured wrist. The WT exercise set was developed by a physical therapist experienced in the treatment of people with wrist fractures and the exercise set had not been used previously. Post-wrist fracture, muscle weakness and instability of the fractured wrist does often persist and can affect every day function [22]. It was therefore thought likely that this type of exercises could motivate people who had sustained a wrist fracture to participate in the study and adhere to the prescribed exercises. Additionally, we wanted the WT group to receive the same attention from a PT as the MST group, therefore the number and duration of supervised sessions were identical in both groups. The WT was conducted using Inimove training equipment (Inimove ApS, Albertslund, Denmark) in supervised training session. Red elastic resistance bands (TheraBand, Akron. $\mathrm{OH})$, a sponge ball and a ball on a plate were used for the home and supervised exercise sessions. All exercises were performed in a sitting position, in order not to stimulate the control of posture simultaneously (Appendix 2). Examples of the WT exercises performed in the present study can be viewed at: https://vimeo.com/252677340/f0bc12ac32.

The training was supervised by two experienced physiotherapists, who were unaware of the results of the subject's baseline measurements.

\section{Outcome measures}

All measurements were conducted at baseline and endpoint of the study by the same blinded physiotherapist. The measurements used in the present study are widely accepted, frequently used and validated. The participants were not informed of the assessment results until completion of the training and all measurements. Questionnaires were completed by participants before and after the training.

\section{Primary outcome measure}

\section{Posturography}

The Sensory Organization Test in the Smart Balance Master (SOT) (Neurocom Inc., Clackamas, OR) was used to measure postural control. The test evaluates the subject's ability to make effective use of somato-sensory, visual and vestibular inputs and suppress inappropriate sensory information. Composite scores of postural sway from the six different sensory conditions were used for analysis. Age-related normative values for the SOT composite scores have been described, ranging from 64 to 70 [23]. Further description of the SOT and the measuring procedure has previously been published [24]. The test has shown moderate-to-good test-retest reliability in older adults [25] and has been validated among people with vestibular disorders [26].

\section{Secondary outcome measures}

\section{Physical function}

Five Times Sit to Stand Test (FTSTS) Functional lower limb muscle strength was measured with the FTSTS [27]. Normative performance values for this test across ages have been reported [28]. The test has displayed discriminative validity, concurrent validity and has been shown to be reliable for comparing lower extremity strength in patients from one visit to the next [29].

10-m Walk Test (10MWT) Gait speed was assessed with the 10MWT. The test was performed at preferred walking speed and repeated at the subject's fastest speed [30]. This test has shown excellent test-retest reliability for comfortable and fastest gait speeds among healthy adults [31].

\section{Sensation in feet}

A biothesiometer electronic device (Model EG electronic BioThesiometer, Newbury, OH, USA) that generated a 120$\mathrm{Hz}$ vibration of varying amplitude (in $\mu \mathrm{m}$ ) was used to measure vibration perception of the plantar surface of the feet. It was applied to the plantar surface of the caput of the first and 
fifth metatarsal bones as well as on the center of the plantar surface of the heel. The biothesiometer has shown excellent reliability in testing vibration perception threshold (VPT) within mild-to-moderate neuropathy [32].

The Semmes-Weinstein pressure aesthesiometer (Semmes-Weinstein Monofilaments, San Jose, USA) (SWM) was used to measure tactile sensitivity. The aesthesiometer comprised 20 nylon filaments of equal length, with varying diameters. The filaments were applied to the plantar surface of the same three points as the biothesiometer. Touch threshold is presented as pressure in grams. Normative values of plantar cutaneous SWM threshold have been described and ranges from 0.4 to $4.0 \mathrm{~g}$, depending on testing sites and age [33]. The SWM test is considered the golden standard to screen for loss of protective sensation [34], which is defined as a level of sensory deficit where a patient can sustain an injury without recognizing a trauma [35]. The test has demonstrated acceptable interrater and intra-rater reliability among healthy adults [36].

For statistical analyses mean values of monofilament and biothesiometer were calculated from individual values measured on plantar surface of heel, caput of the first and fifth metatarsal bones, left and right.

\section{Vestibular function}

Head Shake Test The Head Shake Test was used to assess symmetry of vestibular function. Eye movements were recorded in the supine position with infrared video goggles in place. Based on the recordings, the occurrence of nystagmus and the number of fast eye beats was calculated by a specialist in neurotology. Greater than two or more beats of nystagmus post head shaking was considered positive for vestibular asymmetry [8-10]. The neurotologist was blinded to all recordings both pre- and post-training. The HST, has shown good specificity (82\%) but less sensitivity (45\%) in pooled analysis [37].

Description of the procedures of all the above tests have previously been described [10].

Video-Head Impulse Test (vHIT) vHIT was used to assess the function of the horizontal semi-circular canals by measuring the eye rotation response to an abrupt head rotation in the plane of the lateral semi-circular canals. The main measure of canal adequacy is the ratio of the eye movement response to the head movement stimulus, i.e., the gain of the vestibulo-ocular reflex (VOR). The measurement of the horizontal VOR by the vHIT has previously been described in detail [38]. The vHIT test was performed with a set of ICS impulse video goggles (GN Otometrics, Taastrup, Denmark), with a camera speed of 250 frames, recording motion of the right eye. Subjects were seated and tested in a well-lit room with an eye-level target at a distance of $1 \mathrm{~m}$.
Twenty passive horizontal head turns, both in the right and left directions were performed. The vHIT test has been demonstrated to be a valid clinical tool for testing the function of the horizontal semi-circular canals and simple to use [38, 39].

\section{Anthropometrics}

Body weight and height were measured and body mass index (BMI) was calculated as $\mathrm{kg} / \mathrm{m}^{2}$.

\section{Questionnaires}

Activities-Specific Balance Confidence Scale (ABC) The participants rated their own confidence in 16 activities of daily living on a percentile scale from 0 (no confidence) to 100 (complete confidence) [40]. The scale has demonstrated strong internal-consistency, reliability, and validity when self-administered [41].

Dizziness Handicap Inventory Scale (DHI) Self-perceived handicap resulting from dizziness was assessed with the DHI scale. The scale contains 25 items relating to physical, emotional and functional domains. The range of possible scores on the DHI is $0-100$. The higher the score, the greater the level of self-perceived handicap resulting from dizziness [42]. It has been shown to demonstrate change over the course of rehabilitation [43].

Demographics of the participants were obtained using a questionnaire. They were also asked about the level of weekly physical activity, 12 months prior to participation in the study as well as during the training period. They were not instructed to limit their physical activity in any way during participation in the study.

\section{Data analysis}

Statistical analyses were conducted using SPSS for Windows version 24.0 (SPSS, Chicago, IL, USA) and the level of significance was set at $p<0.05$. Data were checked for normality using the Kolmogorov-Smirnov test. Results are shown as means, standard deviation (SD), median and range. The Mann-Whitney $U$ test was used to compare groups at baseline for not normally distributed variables and independent samples $t$ test for the normally distributed ones.

Improvements over time were calculated using the Wilcoxon test for the not normally or paired samples $t$ test for the normally distributed variables. This was done for each of the training group separately. Effect sizes were calculated according to method described by Cohen for non-parametric variables; effect size $(r)=z$ divided by square root of total 
number of observations [44]. Large magnitude of effect is considered to be: $r \leq 0.50$, medium-sized effects $0.3-0.5$ and small effects $0.1-0.3$.

Univariate general linear models with statistical adjustment for baseline values, gender and age were used to compare endpoint differences between the two training groups.

\section{Results}

\section{Baseline}

The baseline characteristics of participants are shown in Table 1 . The groups were largely comparable, besides vibration sensation, which was significantly poorer in the MST group at baseline $(p=0.02)$.

\section{Drop-out}

In the present study, drop-out rate was $10.6 \%(n=5)$ in the WT group, and $20.8 \%(n=10)$ in the MST group. There were no differences in baseline characteristics between participants who withdrew from the training and those who completed the training in both groups, except that those who stopped in the WT group were older, with a mean age of 70.4 years versus 60.5 years in the MST group.

Participants did not have to provide an explanation for discontinuing training, so data about the reasons for dropping out of the study are limited. However, in the MST group, four dropped out because they felt their balance was fine, three decided to drop out because they did not adhere to the home exercises, two did not arrive for their first training session and one quit because of personal reasons. There was no difference in reporting between the two groups of their exercise adherence.

\section{Intervention}

Table 2 shows within-group mean changes in postural control, foot sensation, vestibular function, perceived dizziness, balance confidence and functional abilities after the intervention. There were significant improvements in both training groups in lower limb muscle strength and SOT. Additionally, there were significant improvements in DHI in the MST group only. The observed effect sizes of significant variables were medium in both groups (MST; SOT: $r=-0.34$, FTSTS: $r=-0.52$, DHI: $r=-0.30 / \mathrm{WT}$; SOT: $r=-0.41$, FTSTS: $r=-0.34$ ).

Table 1 Baseline characteristics of the participants categorized by groups

\begin{tabular}{|c|c|c|c|c|c|c|c|}
\hline \multirow[t]{2}{*}{ Variable } & \multicolumn{3}{|c|}{ Wrist training $(n=42)$} & \multicolumn{3}{|c|}{ Multi-sensory training $(n=38)$} & \multirow[t]{2}{*}{$p$ value* } \\
\hline & Mean \pm SD & Median & Range & Mean \pm SD & Median & Range & \\
\hline Age (years) & $60.8 \pm 6.7$ & 61 & $50-75$ & $62.7 \pm 7.9$ & 63 & $50-75$ & 0.38 \\
\hline Sex; females/males (n) & $36 / 6$ & & & $33 / 5$ & & & \\
\hline BMI $\left(\mathrm{kg} / \mathrm{m}^{2}\right)$ & $28.5 \pm 6.0$ & 27 & $19-59$ & $28.4 \pm 6.2$ & 28 & $19-57$ & 0.97 \\
\hline Phys act prev 12 months (h/week) & $2 \pm 0.8$ & 2 & $1-3$ & $2 \pm 0.6$ & 2.0 & $1-3$ & 0.36 \\
\hline Falls previous 12 months (n) & $2 \pm 1.3$ & 1 & $1-5$ & $2 \pm 1.3$ & 1.0 & $1-6$ & 0.26 \\
\hline Total fractures over lifespan (n) & $2 \pm 1.2$ & 2 & $1-6$ & $2 \pm 1.6$ & 2.0 & $1-8$ & 0.52 \\
\hline 10MWT comfort speed (m/s) & $1.4 \pm 0.19$ & 1.4 & $1.0-1.8$ & $1.4 \pm 0.19$ & 1.4 & $0.7-1.7$ & 0.93 \\
\hline 10MWT fast speed $(\mathrm{m} / \mathrm{s})$ & $1.9 \pm 0.3$ & 1.8 & $1.4-2.7$ & $1.8 \pm 0.30$ & 1.8 & $1.1-2.5$ & 0.42 \\
\hline FTSTS $(s)$ & $11.4 \pm 2.41$ & 11.2 & $5.9-15.9$ & $11.70 \pm 2.61$ & 11.9 & $6.8-19.1$ & 0.50 \\
\hline Monofilament (g) & $1.6 \pm 1.6$ & 1.1 & $0.3-7.7$ & $1.6 \pm 1.1$ & 1.3 & $0.4-4.3$ & 0.46 \\
\hline Biothesiometer $(\mu \mathrm{m})$ & $2.5 \pm 3.8$ & 1.1 & $0.3-20.0$ & $3.6 \pm 4.9$ & 1.8 & $0.6-25.5$ & $\mathbf{0 . 0 2} *$ \\
\hline Head Shake Test ( $\%$ positive $)^{\dagger}$ & $76.2 \%$ & & & $89.5 \%$ & & & 0.12 \\
\hline vHIT left (gain) & $0.9 \pm 0.1$ & 0.9 & $0.8-1.1$ & $0.9 \pm 0.1$ & 0.9 & $0.7-1.2$ & 0.35 \\
\hline vHIT right (gain) & $1 \pm 0.1$ & 1.0 & $0.9-1.3$ & $1.0 \pm 0.2$ & 1.0 & $0.8-1.6$ & 0.99 \\
\hline SOT composite (score) & $72 \pm 7.4$ & 73 & $50-81$ & $74 \pm 7.8$ & 76 & $52-86$ & 0.11 \\
\hline DHI (score) & $9 \pm 14.9$ & 1 & $0-62$ & $13.0 \pm 19.6$ & 4 & $0-66$ & 0.33 \\
\hline $\mathrm{ABC}(\%)$ & $88 \pm 13.1$ & 93 & $43-100$ & $87.0 \pm 13.3$ & 90 & $40-100$ & 0.59 \\
\hline
\end{tabular}

Monofilament and biothesiometer; mean values measured on plantar surface of: heel, caput of the first and fifth metatarsal bones, left and right Significant values are shown in bold

BMI, body mass index, 10MWT $10 \mathrm{~m}$ Walk Test, FTSTS Five Times Sit to Stand Test, vHIT Video Head Impulse Test, SOT Sensory Organization Test, $D H I$ The Dizziness Handicap Inventory, $A B C$ Activities-Specific Balance Confidence Scale

*Difference between groups according to Mann-Whitney $U$ test (not normally distributed variables) and independent samples $t$ test (normally distributed variables)

$\dagger$ Positive HST: $\geq 2$ fast eye beats 
Table 2 Within-group changes in functional ability, postural control, sensation, perceived dizziness and confidence after the interventions

\begin{tabular}{|c|c|c|c|c|c|c|c|c|}
\hline \multirow[t]{2}{*}{ Outcome measure } & \multicolumn{4}{|c|}{ Wrist training $(n=42)$} & \multicolumn{4}{|c|}{ Multi-sensory training $(n=38)$} \\
\hline & $\Delta$ & $95 \% \mathrm{CI}$ & & $p$ value* & $\Delta$ & $95 \% \mathrm{CI}$ & & $p$ value* \\
\hline SOT composite (score) & 3.6 & 1.363 & 5.813 & $<0.01 *$ & 4.2 & 1.495 & 6.943 & $<0.01 *$ \\
\hline Monofilament (g) & -0.2 & -0.465 & -0.012 & 0.14 & -0.1 & -0.360 & 0.222 & 0.08 \\
\hline Biothesiometer $(\mu \mathrm{m})$ & -0.3 & -0.697 & 0.133 & 0.11 & -0.2 & -0.629 & 0.179 & 0.38 \\
\hline Head Shake Test positive ${ }^{\mathrm{a}}(\%)$ & 0.0 & -0.195 & 0.195 & 1.0 & -15.8 & -0.320 & 0.005 & 0.06 \\
\hline vHIT left (gain) & 0.01 & -0.012 & 0.037 & 0.30 & 0.0 & -0.011 & 0.045 & 0.23 \\
\hline vHIT right (gain) & 0.0 & -0.042 & 0.005 & 0.15 & 0.0 & -0.066 & 0.027 & 0.41 \\
\hline 10MWT comfort speed $(\mathrm{m} / \mathrm{s})$ & 0.0 & -0.025 & 0.063 & 0.39 & 0.0 & -0.032 & 0.064 & 0.51 \\
\hline 10MWT fast speed (m/s) & 0.0 & -0.037 & 0.082 & 0.45 & 0.1 & -0.002 & 0.094 & 0.06 \\
\hline FTSTS (s) & -1.0 & -1.537 & -0.444 & $<0.01 *$ & -1.5 & -1.964 & -0.996 & $<0.001 *$ \\
\hline DHI (score) & -2.3 & -5.673 & 1.088 & 0.21 & -4.7 & -8.283 & -1.086 & $0.01 *$ \\
\hline $\mathrm{ABC}$ (score) & 0.9 & -1.152 & 2.935 & 0.98 & 2.3 & -0.141 & 4.743 & 0.69 \\
\hline
\end{tabular}

Monofilament and biothesiometer; mean values measured on plantar surface of: heel, caput of the first and fifth metatarsal bones, left and right

Significant values are shown in bold

$\Delta$ mean change, SOT Sensory Organization Test, 10MWT $10 \mathrm{~m}$ Walk Test, FTSTS Five Times Sit to Stand Test, DHI The Dizziness Handicap Inventory, ABC Activities-Specific Balance Confidence Scale, vHIT Video Head Impulse Test

*Baseline - endpoint differences: Wilcoxon non-parametric test and paired samples $t$ test

apositive HST: $\geq 2$ fast eye beats
Weekly physical activity level during the training period, decreased by $0.02 \mathrm{~h} /$ week in the WT group and increased by $0.2 \mathrm{~h} /$ week in the MST group, compared to physical activity level 12 months prior to participation in the study. According to Mann-Whitney $U$ test, baseline - endpoint differences in physical activity levels between the groups, were not significantly different $(p=0.11)$.

According to linear models, correcting for baseline values, age and gender (Table 3), there was a significant in between group difference in endpoint SOT $(\mathrm{MST}+3.1$, $p=0.01$ ), but not in other outcome variables.

\section{Participants with poor baseline SOT}

When looking at within-group changes in participants who had SOT baseline composite scores below age norms, we found that more outcome measures improved in the MST than in the WT group. The observed effect sizes of significant variables in the MST were large $(r=-0.64)$ for all the variables. However, the number of cases was small (MST: $n=5$, WT: $n=8$ ) and statistical power was limited (Table 4). In a separate comparison between groups, using linear regression corrected for baseline values (not shown in a table), we found that the MST had a higher endpoint SOT than the WT group $(+7.4, p=0.012)$.

There were a few outliers in the data set; seven people had 5-6 falls within the previous 12 months, four had DHI scores of $>54$ [42] and two had ABC scores of $<50$ [45].
Table 3 Endpoint differences between groups in functional ability, sensation, postural control, perceived dizziness and confidence

\begin{tabular}{lrrrl}
\hline Outcome measure & \multicolumn{1}{l}{$B$} & $95 \%$ CI & & $p$ value \\
\hline SOT composite (score) & 3.095 & 0.797 & 5.393 & $\mathbf{0 . 0 1} *$ \\
Monofilament $(\mathrm{g})$ & 0.134 & -0.158 & 0.426 & 0.36 \\
Biothesiometer $(\mu \mathrm{m})$ & 0.250 & -0.139 & 0.639 & 0.20 \\
Number fast eye beats & 0.135 & 0.382 & 0.950 & 0.88 \\
vHIT left (gain) & 0.006 & -0.025 & 0.037 & 0.71 \\
vHIT right (gain) & 0.002 & -0.037 & 0.040 & 0.93 \\
10MWT comfort speed (m/s) & 0.002 & -0.056 & 0.060 & 0.94 \\
10MWT fast speed (m/s) & 0.012 & -0.056 & 0.081 & 0.73 \\
FTSTS (s) & -0.463 & -1.184 & 0.258 & 0.20 \\
DHI (score) & -0.571 & -4.079 & 2.938 & 0.75 \\
ABC (score) & 1.303 & -1.318 & 3.924 & 0.33 \\
\hline
\end{tabular}

Monofilament; mean values measured on plantar surface of: heel, caput of the first and fifth metatarsal bones, left and right

Significant values are shown in bold

SOT Sensory Organization Test, IOMWT $10 \mathrm{~m}$ Walk Test, FTSTS Five Times Sit to Stand Test, DHI The Dizziness Handicap Inventory, $A B C$ Activities-Specific Balance Confidence Scale, $v H I T$ Video-Head Impulse Test

*Results show MST compared to WT based on univariate general linear models which corrected for base line values, age and gender

The analysis of data was also conducted without the outliers in the analyses, but their removal did not make a difference in the results of the study (data not shown). 
Table 4 Within-group changes in functional ability, pressure plantar sensation and postural control after the intervention, among participants with SOT baseline values below age norms

\begin{tabular}{|c|c|c|c|c|c|c|c|c|}
\hline \multirow[t]{2}{*}{ Outcome measures } & \multicolumn{4}{|c|}{ Wrist training $(n=8)$} & \multicolumn{4}{|c|}{ Multi-sensory training $(n=5)$} \\
\hline & $\Delta$ & $95 \% \mathrm{C}$ & & $p$ value* & $\Delta$ & $95 \% \mathrm{C}$ & & $p$ value* \\
\hline SOT composite (score) & 9.5 & 5 & 14 & 0.01* & 16.8 & 12.1 & 21.5 & $0.04 *$ \\
\hline Monofilament (g) & 0.1 & -0.2 & 0.4 & 0.31 & -0.4 & -1.2 & 0.4 & $0.04 *$ \\
\hline 10MWT fast speed $(\mathrm{m} / \mathrm{s})$ & 0.0 & 0.0 & 0.1 & 0.12 & 0.1 & 0.0 & 0.2 & $0.04 *$ \\
\hline FTSTS (s) & -1.1 & -3.0 & 0.8 & 0.16 & -2.9 & -4.7 & -1.1 & $0.04 *$ \\
\hline
\end{tabular}

Monofilament; mean values measured on plantar surface of: heel, caput of the first and fifth metatarsal bones, left and right

Significant values are shown in bold

SOT composite age norms (scores): $20-59$ years: $\geq 70 ; 60-69$ years: $\geq 68 ; 70-79$ years: $\geq 64$

$\Delta$ mean change, SOT Sensory Organization Test composite scores, 10MWT $10 \mathrm{~m}$ Walk Test, FTSTS Five Times Sit to Stand Test

*Baseline - endpoint differences: Wilcoxon non-parametric test

\section{Discussion}

The aim of the present study was to investigate whether MST improves postural control, vestibular function, tactile sensation and functional ability among people with fall-related wrist fractures compared to those receiving WT. In a direct comparison, we found that the MST group displayed significantly higher scores on the SOT at the end of the study than WT. Considering within-group changes during the intervention, a significant improvement on DHI was only observed in the MST group but not the WT group. However, significant improvements in both groups were observed for the FTSTS and SOT. According to our results, poor balance control at baseline was associated with a better improvement in postural control during the intervention.

These modest findings were somewhat unexpected, because in a previous pilot study [18], MST resulted in greater improvements in postural control, functional ability and confidence in activities of daily living. This difference in outcomes can possibly be explained by different study populations. The participants in the pilot study were older, between 70 and 92 years, had sustained multiple falls and fractures, most of them had decreased sensation in their lower limbs and they had numerous comorbidities. They were physically weaker, more unstable and less confident during daily activities, as demonstrated by their poorer performance in the different tests and questionnaire (ABC scale) at baseline. The participants in the present study were younger, 50-75 years of age and retrospectively quite healthy and well-functioning. Although, the prevalence of vestibular asymmetry was high (83\%) among the wrist fracture participants and $35 \%$ of them had reduced plantar sensitivity [33], they were physically active and not complaining of dizziness or unsteadiness as demonstrated by their low DHI and high $\mathrm{ABC}$ scores. The balance performance for $84 \%$ of them was within normal age range, as measured by the SOT [23]. Walking speed [46] and lower limb functional muscle strength [47] were within normal age-related ranges for healthy individuals among all of the participants and vibration sense [48] was within the normal range for $89 \%$ of them. As the participants in the present study were healthy and generally in good physical condition, this might have made it more difficult to achieve improvements with the MST training. Subgroup analysis with wrist fracture participants (WT $n=8$; MST $n=5$ ) with below normal baseline SOT composite scores support this. Reduced postural control at baseline was associated with a better improvement in postural control during the intervention. Additionally, the effects of the intervention among participants with reduced postural control at baseline demonstrated that the MST resulted in significant better outcomes than the WT for these participants. The WT group showed a mean change of 9.5 composite scores on the SOT, which is close to a learning effect ( 8 scores) due to repeated measurements [49]. However, the MST group exceeded that with mean change of 16.8 scores. Fast walking speed increased by $0.1 \mathrm{~m} / \mathrm{s}$ post-training among the MST participants, which is regarded clinically meaningful [50], but no change was observed in the WT group. Tactile sensitivity improved as well only in the MST group. Although minimal clinically important differences in tactile sensitivity have not been reported, it has been shown that reduced tactile sensitivity is associated with fall-related wrist fractures [10]. Additionally, a clinically meaningful change of $2.9 \mathrm{~s}$ was reached in the FTSTS [51] in the MST group. These results imply that the MST was more effective than the WT among people with reduced postural control. However, as the number of participants with reduced postural control at baseline was very small, these findings need to be confirmed using a larger sample size before firm conclusions can be drawn.

Furthermore, the study protocols in the pilot study and the present study were different which can as well explain the limited improvements observed in the present study. The number of training sessions in the pilot study was dictated 
by the Icelandic social security reimbursement rules. Each referral included 20 reimbursed sessions, of which two were used for pre- and post-assessments and the remaining 18 for training. The frequency of supervised training sessions in the present study was lower than in the pilot study, consisting of only six supervised sessions and prescribed home exercises. This reduction in supervised sessions was based on clinical experience, where six supervised sessions of the MST and daily home exercises have led to decreased dizziness and improved postural stability among people with unilateral and bilateral peripheral vestibular hypofunction. This approach is as well in line with the clinical practice guidelines from the American Physical Therapy Association for vestibular rehabilitation for peripheral vestibular hypofunction [13]. According to these guidelines, based on expert opinion, persons with chronic unilateral vestibular hypofunction may need supervised sessions once a week for 4-6 weeks, together with daily home exercises.

The drop-out rate in the present study was $11 \%$ in the WT group, and $21 \%$ in the MST group, which can be considered acceptable in a randomized controlled trial [52].

One variable of interest in this study was asymmetric vestibular function, which has been shown to be associated with falls and wrist fractures $[8,10]$. Post-training, there were no significant changes observed on the vHIT in the training groups. The gain of the vestibulo-ocular reflex (VOR) as measured with the vHIT, was within normative values [53] at baseline among the participants so there was most likely a ceiling effect. Conversely, there was a $16 \%$ borderline significant $(p=0.06)$ reduction of vestibular asymmetry in the MST group as measured by the Head Shake Test but no change was observed in the group receiving the WT. Previously, Hanson et al. found an $18.5 \%$ reduction of vestibular asymmetry after 9 weeks of group sessions two times/week of vestibular rehabilitation among persons post wrist fracture [11]. The reduction in vestibular asymmetry post-training in our study indicates that the MST can positively affect asymmetric vestibular function. This is of importance with regards to fall prevention, as vestibular asymmetry disturbs fall-prevention movements which become smaller or distorted leading to increased danger of falling and imbalance [54]. However, as our findings did not reach statistical significance, no firm conclusions on the effect of the MST on vestibular asymmetry can be drawn from this study.

\section{Strength and limitations}

To the best of our knowledge, no previous studies have investigated the effect of MST among people with fall-related wrist fractures compared to WT. However, it is a limitation that the participants were healthy and well-functioning in accordance with relatively young age thus potentially masking the true potential of the MST.

As in every intervention study, compliance is important. Even though the participants did complete an exercise diary, their adherence to the home exercises cannot be verified.

\section{Conclusion}

MST improves postural control among people who have sustained a fall-related wrist fracture. The results of the study further suggest that the program is more effective for those with balance scores below age-related norms on the sensory organization test.

Acknowledgements The authors would like to thank the people with a fall-related wrist fracture, who gave up time to participate in the trial and the physical therapists, who delivered the trial interventions; Holmfridur H. Sigurdardóttir (MST) and Anne S. Melén (WT).

Funding This study was funded by the St. Josef's Hospital Fund (SJHF), Reykjavik, Iceland, the Icelandic Physiotherapy Association Research Fund (IPARF), the Landspítali Háskólasjúkrahús (Landspitali University Hospital) Research Fund (A-2014-076, A-2017-061, A-2018-067), the Icelandic Gerontological Society Research Fund (IGSRF) and the Helga Jónsdóttir and Sigurlidi Kristjánsson Geriatric Research Fund (HJSKGRF).

\section{Compliance with ethical standards}

Conflict of interest All authors declare that they have no conflicts of interest.

Statement of human and animal rights This study received permission from the Icelandic National Bioethics Committee (VSNb2013110036/03.11). All procedures performed in studies involving human participants were in accordance with the ethical standards of the institutional and/or national research committee and with the 1964 Helsinki declaration and its later amendments or comparable ethical standards.

Informed consent Informed consent was obtained from all individual participants included in the study.

OpenAccess This article is distributed under the terms of the Creative Commons Attribution 4.0 International License (http://creativeco mmons.org/licenses/by/4.0/), which permits unrestricted use, distribution, and reproduction in any medium, provided you give appropriate credit to the original author(s) and the source, provide a link to the Creative Commons license, and indicate if changes were made.

\section{Appendix 1: Multi-sensory training}

Duration of home exercises: minimum $15 \mathrm{~min}, 5-7$ times each week. 
Exercises are performed with bare feet.

Two sets of each exercise are performed. Rest interval between sets, 10-15 s, or until symptoms of dizziness or unsteadiness have subsided.

Throughout all the exercises, focus is on weight distribution on the soles of the feet in order to recognize and control the position and movements of the body.

\section{Proprioceptive training}

Standing. Exercises performed with eyes open and then closed:

1. Weight shift from side to side, 4-6 times in each direction.

2. Weight shift forwards and backwards, 4-6 times in each direction.

Perform exercise (1) and (2) slowly, using smooth corrective motions at the ankles and avoid high-frequency movements at the hips and upper body.

3. Stamping feet on the spot, 7-10 times.

Standing on a balance cushion, foam or trampoline.

Exercises 1-3 repeated.

Walking (preferred walking speed). 2 min for each exercise.

4. Paying attention to weight distribution on the feet.

5. Stamping feet.

6. Walking on uneven surfaces and surfaces with different textures.

\section{Vestibular and eye control training}

Each of exercises 7-21 are performed until symptoms of dizziness and/or unsteadiness are provoked.

Standing.

7. Eyes kept still during movements of the head in all directions.

8. Moving the head in all directions, eyes following head movements.

9. Moving the head in all directions with eyes closed.

10. Quick movement of the head in all directions with fixed gaze.

11. Quick movement of the head in all directions, eyes following head movements.
12. Quick movement of the head in all directions with eyes closed.

Sitting on a rotational chair.

13. Chair rotated irregularly in both directions with eyes open and closed.

Standing on one foot or on a turning disc.

14. Quick right and left turns, eyes open and closed.

On trampoline.

15. Walking and bouncing.

\section{Combined proprioceptive and vestibular training}

Standing on a balance cushion, foam or trampoline.

Exercises 7-12.

16. Reaching for an object in different directions.

17. Catching and throwing a ball.

18. Keeping a balloon in the air.

Walking (preferred walking speed).

Exercises 17-18

19. Moving head in all directions, fixing gaze on surrounding objects.

20. Quick right and left turns.

Sitting on a rotational chair or standing on a turning disc.

21. Chair/disc rotated irregularly in both directions while reading text.

\section{Fall reaction training}

Each of exercises 22-24 are performed 4-6 times in each direction.

Standing.

22. Practicing quick stepping actions in different directions to prevent a fall. 
23. Subjects pushed in different directions with and without prior warning.

Walking (preferred walking speed).

24. Subject pushed irregularly in different directions with and without prior warning.

Training method instigated by:

Dr. Ella K. Kristinsdottir, PT, PhD

Bergthora Baldursdottir, PT, MSc

\section{Appendix 2: Wrist stabilization training}

Duration of home exercises: minimum $15 \mathrm{~min}, 5-7$ times each week.

All exercises are performed in the sitting position.

\section{Exercises using a golf ball on a plate ( $5 \mathrm{~min}$ )}

1. Try to keep the golf ball steady in the center of the plate.

2. Try to move the ball slightly without touching the rim of the plate.

3. Move the forearm away from your body while keeping the ball steady in the middle of the cup.

\section{Exercises using a red elastic band ( 5 min)}

Grab the elastic band using both hands, elbows against the side of the body.

1. Elbows flexed to $90^{\circ}$. Pull the elastic band to the side using the fractured arm resist the pull with the non-fractured arm.

2. One elbow is flexing and the opposite elbow is extending. Pull the elastic band using the fractured forearm by bending the elbow upwards and stabilizing with the non-fractured forearm against the thigh and then resist the forces in the opposite direction.

It is important to maintain the wrist in neutral or slightly extended throughout the exercises.

\section{Exercises using a sponge ball ( $5 \mathrm{~min})$}

Squeeze a sponge ball as tightly as possible, count to three and relax, repeat ten times. Rest for a moment (10-15 s) and fully extend the fingers. Repeat the exercise set three times.

\section{References}

1. Gillespie LD, Robertson MC, Gillespie WJ et al (2012) Interventions for preventing falls in older people living in the community. Cochrane Database Syst Rev. https://doi.org/10.1002/14651858. CD007146.pub3

2. Nordell E, Jarnlo GB, Jetsen C et al (2000) Accidental falls and related fractures in 65-74 year olds: a retrospective study of 332 patients. Acta Orthop Scand 71:175-179

3. Cuddihy MT, Gabriel SE, Crowson CS et al (1999) Forearm fractures as predictors of subsequent osteoporotic fractures. Osteoporos Int 9:469-475

4. Johnson NA, Stirling ER, Divall P et al (2017) Risk of hip fracture following a wrist fracture-a meta-analysis. Injury 48:399-405

5. Braithwaite RS, Col NF, Wong JB (2003) Estimating hip fracture morbidity, mortality and cost. J Am Geriatr Soc 51:364-370

6. Edwards BJ, Song J, Dunlop DD et al (2010) Functional decline after incident wrist fractures-study of osteoporotic fractures: prospective cohort study. Bmj 341:c3324

7. Nordell E, Kristinsdottir EK, Jarnlo GB et al (2005) Older patients with distal forearm fracture. A challenge to future fall and fracture prevention. Aging Clin Exp Res 17:90-95

8. Kristinsdottir EK, Nordell E, Jarnlo G-B et al (2001) Observation of vestibular asymmetry in a majority of patients over 50 years with fall-related wrist fractures. Acta Otolaryngol 121:481-485

9. Kristinsdottir EK, Jarnlo G-B, Magnusson M (2000) Asymmetric vestibular function in the elderly might be a significant contributor to hip fractures. Scand J Rehabil Med 32:56-60

10. Baldursdottir B, Petersen H, Jonsson PV et al (2018) Sensory impairments and wrist fractures: a case-control study. J Rehabil Med 50:209-215

11. Ekvall Hansson E, Dahlberg LE, Magnusson M (2015) Vestibular rehabilitation affects vestibular asymmetry among patients with fall-related wrist fractures - a randomized controlled trial. Gerontology 61:310-318

12. Whitney SL, Alghwiri AA, Alghadir A (2016) An overview of vestibular rehabilitation. Handb Clin Neurol 137:187-205

13. Hall CD, Herdman SJ, Whitney SL et al (2016) Vestibular rehabilitation for peripheral vestibular hypofunction: an evidence-based clinical practice guideline: From the American physical therapy association neurology section. J Neurol Phys Ther 40:124-155

14. Hu MH, Woollacott MH (1994) Multisensory training of standing balance in older adults: I. Postural stability and one-leg stance balance. J Gerontol 49:M52-M61

15. Hu MH, Woollacott MH (1994) Multisensory training of standing balance in older adults: II. Kinematic and electromyographic postural responses. J Gerontol 49:M62-M71

16. Alfieri FM, Riberto M, Gatz LS et al (2012) Comparison of multisensory and strength training for postural control in the elderly. Clin Interv Aging 7:119-125

17. Alfieri FM, de Jesus Guirro RR, Teodori RM (2010) Postural stability of elderly submitted to multisensorial physical therapy intervention. Electromyogr Clin Neurophysiol 50:113-119

18. Kristinsdottir EK, Baldursdottir B (2014) Effect of multi-sensory balance training for unsteady elderly people: pilot study of the "Reykjavik model". Disabil Rehabil 36:1211-1218

19. Handoll HH, Elliott J (2015) Rehabilitation for distal radial fractures in adults. Cochrane Database Syst Rev. https://doi. org/10.1002/14651858.CD003324.pub3

20. Bruder AM, Shields N, Dodd KJ et al (2016) A progressive exercise and structured advice program does not improve activity more than structured advice alone following a distal radial fracture: a multi-centre, randomised trial. J Physiother 62:145-152

21. Hebert JR, Corboy JR, Manago MM et al (2011) Effects of vestibular rehabilitation on multiple sclerosis-related fatigue and 
upright postural control: a randomized controlled trial. Phys Ther 91:1166-1183

22. Bruder AM, Taylor NF, Dodd KJ et al (2013) Physiotherapy intervention practice patterns used in rehabilitation after distal radial fracture. Physiotherapy 99:233-240

23. NeuroCom (2000) Operator's manual: SMART balance master, principles of operation. NeuroCom International Inc, Clackamas

24. Buatois S, Gueguen R, Gauchard G et al (2006) Posturography and risk of recurrent falls in healthy non-institutionalized persons aged over 65 . Gerontology 52:345-352

25. Ford-Smith CD, Wyman JF, Elswick RK et al (1995) Test-retest reliability of the sensory organization test in noninstitutionalized older adults. Arch Phys Med Rehabil 76:77-81

26. Whitney SL, Marchetti GF, Schade AI (2006) The relationship between falls history and computerized dynamic posturography in persons with balance and vestibular disorders. Arch Phys Med Rehabil 87:402-407

27. Lord SR, Murray SM, Chapman K et al (2002) Sit-to-stand performance depends on sensation, speed, balance, and psychological status in addition to strength in older people. J Gerontol A Biol Sci Med Sci 57:M539-M543

28. Bohannon RW, Bubela DJ, Magasi SR et al (2010) Sit-to-stand test: performance and determinants across the age-span. Isokinet Exerc Sci 18:235-240

29. Whitney SL, Wrisley DM, Marchetti GF et al (2005) Clinical measurement of sit-to-stand performance in people with balance disorders: validity of data for the Five-Times-Sit-to-Stand Test. Phys Ther 85:1034-1045

30. Perera S, Mody SH, Woodman RC et al (2006) Meaningful change and responsiveness in common physical performance measures in older adults. J Am Geriatr Soc 54:743-749

31. Bohannon RW (1997) Comfortable and maximum walking speed of adults aged 20-79 years: reference values and determinants. Age Ageing 26:15-19

32. van Deursen RW, Sanchez MM, Derr JA et al (2001) Vibration perception threshold testing in patients with diabetic neuropathy: ceiling effects and reliability. Diabet Med 18:469-475

33. Rinkel WD, Aziz MH, Van Deelen MJ et al (2017) Normative data for cutaneous threshold and spatial discrimination in the feet. Muscle Nerve 56:399-407

34. Craig AB, Strauss MB, Daniller A et al (2014) Foot sensation testing in the patient with diabetes: introduction of the quick and easy assessment tool. Wounds 26:221-231

35. Bus SA, van Netten JJ, Lavery LA et al (2016) Screening for diabetic peripheral neuropathy: IWGDF guidance on the prevention of foot ulcers in at-risk patients with diabetes. Diabetes Metab Res Rev 32:16-24

36. Snyder BA, Munter AD, Houston MN et al (2016) Interrater and intrarater reliability of the Semmes-Weinstein monofilament 4-2-1 stepping algorithm. Muscle Nerve 53:918-924

37. Dros J, Maarsingh OR, van der Horst HE et al (2010) Tests used to evaluate dizziness in primary care. CMAJ 182:E621-E631

38. MacDougall HG, Weber KP, McGarvie LA et al (2009) The video head impulse test: diagnostic accuracy in peripheral vestibulopathy. Neurology 73:1134-1141

39. Weber KP, MacDougall HG, Halmagyi GM et al (2009) Impulsive testing of semicircular-canal function using video-oculography. Ann N Y Acad Sci 1164:486-491
40. Powell LE, Myers AM (1995) The Activities-Specific Balance Confidence (ABC) Scale. J Gerontol A Biol Sci Med Sci 50A:M28-M34

41. Talley K, Wyman J, Gross C (2008) Psychometric properties of the Activities-Specific Balance Confidence Scale and the survey of activities and fear of falling in older women. J Am Geriatr Soc $56: 328-333$

42. Jacobson GP, Newman CW (1990) The development of the Dizziness Handicap Inventory. Arch Otolaryngol Head Neck Surg 116:424-427

43. Serbetcioglu B, Mutlu B (2008) Vestibular rehabilitation outcome of patients with unilateral vestibular deficits. Mediterr J Otol 4:24-31

44. Cohen J (1988) Statistical power analysis for the behavioral sciences, 2nd edn. Erlbaum, Hillsdale

45. Myers A, Fletcher P, Myers A et al (1998) Discriminative and evaluative properties of the Activities-Specific Balance Confidence (ABC) Scale. J Gerontol A Biol Sci Med Sci 53:M287-M294

46. Fritz S, Lusardi M (2009) White paper: "walking speed: the sixth vital sign". J Geriatr Phys Ther 32:46-49

47. Meretta BM, Whitney SL, Marchetti GF et al (2006) The five times sit to stand test: responsiveness to change and concurrent validity in adults undergoing vestibular rehabilitation. J Vestib Res 16:233-243

48. Maffei L, Premrou V, Roldan P et al (2014) Vibration perception threshold in the screening of sensorimotor distal symmetric polyneuropathy: the need of more accurate age-specific reference values. J Diabetes Sci Technol 8:621-622

49. Wrisley DM, Stephens MJ, Mosley S et al (2007) Learning effects of repetitive administrations of the sensory organization test in healthy young adults. Arch Phys Med Rehabil 88:1049-1054

50. Palombaro KM, Craik RL, Mangione KK et al (2006) Determining meaningful changes in gait speed after hip fracture. Phys Ther 86:809-816

51. Bohannon RW (2006) Reference values for the five-repetition sit-to-stand test: a descriptive meta-analysis of data from elders. Percept Mot Skills 103:215-222

52. Cramer H, Haller H, Dobos G et al (2016) A systematic review and meta-analysis estimating the expected dropout rates in randomized controlled trials on yoga interventions. Evid Based Complement Altern Med. https://doi.org/10.1155/2016/5859729

53. McGarvie LA, MacDougall HG, Halmagyi GM et al (2015) The Video Head Impulse Test (vHIT) of semicircular canal functionage-dependent normative values of VOR gain in healthy subjects. Front Neurol 6:154

54. Allum JH, Carpenter MG, Honegger F et al (2002) Age-dependent variations in the directional sensitivity of balance corrections and compensatory arm movements in man. J Physiol 542:643-663

Publisher's Note Springer Nature remains neutral with regard to jurisdictional claims in published maps and institutional affiliations. 